\title{
Global Asymptotic Stability of the Limit Cycle in Piecewise Linear versions of the Goodwin Oscillator
}

\author{
Adrián A. Salinas-Varela, Guy-Bart Stan, Jorge Gonçalves \\ Department of Engineering, University of Cambridge. \\ email: $\{$ aas47, gvs22, jmg777\}@cam.ac.uk
}

\begin{abstract}
Conditions in the form of linear matrix inequalities (LMIs) are used in this paper to guarantee the global asymptotic stability of a limit cycle oscillation for a class of piecewise linear (PWL) systems defined as the feedback interconnection of a saturation controller with a single input, single output (SISO) linear time-invariant (LTI) system. The proposed methodology extends previous results on impact maps and surface Lyapunov functions to the case when the sets of expected switching times are arbitrarily large. The results are illustrated on a PWL version of the Goodwin oscillator.
\end{abstract}

\section{INTRODUCTION}

Motivated by the pervasiveness of dynamical systems that exhibit stable limit cycle oscillations (see Goldbeter [1996], Mosekilde [1997], and Strogatz [2003] for numerous examples), this paper focuses on the global analysis of limit cycle oscillations of piecewise linear (PWL) systems. Such systems are defined by a set of affine linear systems

$$
\dot{x}=A_{\sigma} x+B_{\sigma}
$$

where $x \in \mathbb{R}^{n}$ is the state, together with a piecewise constant rule to switch among them

$$
\sigma(x) \in\{1, \ldots, M\}
$$

that depends on present values (and possibly also on past values) of $x$. This kind of systems are typically used to model processes which encompass several modes of operation with a different (but linear) dynamical behaviour in each mode. Unfortunately, the majority of the analytical tools devised specifically for this kind of systems - whether based on extensions of Lyapunov's theory (e.g. Branicky [1998], Johansson and Rantzer [1998], Petterson and Lennartson [2002], Prajna and Papachristodoulou [2003]) or not (e.g Margaliot [2006], Iwatani and Hara [2006], Xu and Antsaklis [1999], Boscain [2002]) - is incompatible with the study of limit cycle oscillations. This apparent limitation was addressed by Gonçalves [2000]. In the latter reference, the stability analysis of a PWL system's limit cycle is achieved via the use of linear matrix inequalities (LMIs) to construct Lyapunov functions on the switching surfaces (the subsets of the state space in which the system is allowed to switch from one mode of operation to another). Unlike the phase plane methods and Poincaré-Bendixson theorem, this methodology generalises easily to high dimensional systems. It has been successfully applied to the global analysis of oscillations in relay feedback systems [Gonçalves, 2000, chapter 5] and to the characterisation of regions of stability of limit cycles for more general PWL systems in Gonçalves [2005]. Nevertheless, the construction of the Lyapunov functions depends upon the impact maps (maps from one switching surface to another), which are in turn characterised by their associated switching times (the time between switches). Consequently, it is impracticable when such switching times are arbitrarily large. This paper is devoted to the analysis of a specific class of systems in which such a situation arises.

\footnotetext{
* This work was supported in part by the Mexican Council of Science and Technology (CONACyT), the Cambridge Overseas Trust and the Overseas Research Studentships Programme.
}

This paper is organised as follows. Section 2 introduces the class of systems that we intend to analyse. Section 3 starts by stating the conditions which guarantee the asymptotic stability of the system's limit cycle in the entirety of its state space with the exception of its stable manifold. Since the presence of this stable manifold implies the existence of arbitrarily large switching times, conditions to overcome such obstacle are then presented. A PWL version of the Goodwin oscillator is used in Section 4 to illustrate our results. Section 5 concludes and gives directions for future research.

\section{PROBLEM DEFINITION}

Consider the feedback interconnection of a SISO LTI system satisfying the following linear dynamic equations

$$
\begin{aligned}
& \dot{x}=A x+B u \\
& y=C x
\end{aligned}
$$

where $x \in \mathbb{R}^{n}$, with a saturation controller defined as

$$
u(t)= \begin{cases}\kappa & \text { if } y(t)<-d \\ -\kappa / d y(t) & \text { if }|y(t)| \leq d \\ -\kappa & \text { if } y(t)>d\end{cases}
$$

where $d>0$ and $\kappa>0$.

By a solution of (3) - (4) we mean functions $(x(t), y(t), u(t))$ satisfying (3) - (4).

As discussed in [Gonçalves, 2000, chapter 7.2], this system is symmetric around the origin and has a unique solution for any initial state. In the state space, the saturation controller introduces two switching surfaces consisting of hyperplanes of dimension $n-1$ :

$$
S:=\left\{x \in \mathbb{R}^{n} \mid C x=d\right\}, \quad \underline{S}:=\left\{x \in \mathbb{R}^{n} \mid C x=-d\right\}
$$

On one side of the switching surface $S(C x>d)$, the system is governed by $\dot{x}=A x-B \kappa$. In between the two switching surfaces $(|C x| \leq d)$, the system is given by $\dot{x}=(A-(\kappa / d) B C) x$. Finally, on the other side of $\underline{S}$ $(C x<-d)$, the system is governed by $\dot{x}=A x+B \kappa$. Define the subsets $S_{+}$and $S_{-}$of $S$ as follows:

$$
\begin{aligned}
& S_{+}:=\{x \in S \mid C(A-(\kappa / d) B C) x \geq 0\} \\
& S_{-}:=\{x \in S \mid C(A-(\kappa / d) B C) x \leq 0\}
\end{aligned}
$$

As shown in Figure $1(\mathrm{~b}), S_{+}\left(S_{-}\right)$is the set of points in $S$ that can be reached by trajectories of $(3)-(4)$ when governed by the subsystem $\dot{x}=(A-(\kappa / d) B C) x$ $(\dot{x}=A x-B \kappa)$. Define also $\underline{S}_{+}:=-S_{+}$and $\underline{S}_{-}:=-S_{-}$, where $x_{s} \in-S$ denotes $x_{s} \in\{x \mid-x \in S\}$.

\subsection{Class of systems under consideration.}

Systems of the form (3) - (4) are nonlinear and as such, they can exhibit extremely complex behaviours. Some may 


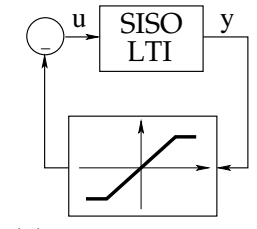

(a) Saturation system

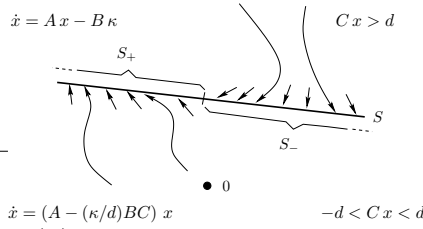

(b) The sets $S_{+}, S_{-} \subset S$

\section{Fig. 1. Problem definition}

be chaotic; others may have have several isolated equilibria; others might have limit cycles, or even some combination of all these behaviours. Here we consider a class of systems which satisfies the following conditions:

- There exists a periodic solution with 4 switches per cycle, i.e. there exists a set of time instants $\left\{t_{1}^{*}, t_{2}^{*}, t_{3}^{*}, t_{4}^{*}\right\}$ which complies with [Gonçalves, 2000, Proposition 3.2].

- The periodic solution is locally stable, i.e. the conditions in [Gonçalves, 2000, Proposition 3.3] are satisfied.

- The SISO LTI system (3) is of dimension 3.

- The matrix $A$ in (3) is Hurwitz.

- The inequality $\kappa C A^{-1} B \leq d$ holds, ensuring that the origin is the only equilibrium point.

- The matrix $(A-(\kappa / d) B C)$ is assumed to have a real negative eigenvalue and a pair of complex conjugate ones with positive real parts.

The last condition implies that the unstable equilibrium $x=0$ possesses a stable manifold, which we will denote as $\mathcal{M}_{s}$. It is readily seen that solutions of (3) - (4) belonging to $\mathcal{M}_{s}$ will approach the origin asymptotically. But what happens when this is not the case? Will all other solutions eventually converge to the limit cycle described by the first two conditions? This is what we understand as "global asymptotic stability of the limit cycle" and constitutes the question that we propose to answer.

\subsection{Structural description.}

The following discussion examines the implications entailed by the assumption regarding the eigenvalues of $(A-(\kappa / d) B C)$. This will play an important role when verifying a set of sufficient conditions which guarantee that the limit cycle is asymptotically stable in $\mathbb{R}^{3} \backslash \mathcal{M}_{s}$.

Let $\mathcal{B}$ be a basis of $\mathbb{R}^{3}$. Suppose the linear map $f: \mathcal{B} \rightarrow \mathcal{B}$ is represented by the matrix $(A-(\kappa / d) B C)$ and denote its eigenvalues as $\lambda_{1,2}=\alpha \pm j \beta$ and $\lambda_{3}=\gamma$. Since $(A-(\kappa / d) B C)$ is real, there exists a real nonsingular matrix $T$ such that $A_{1}:=T^{-1}(A-(\kappa / d) B C) T$ is in real Jordan form [Horn and Johnson, 1985, section 3.4]. In other words,

$$
A_{1}:=T^{-1}(A-(\kappa / d) B C) T=\left[\begin{array}{ccc}
\alpha & -\beta & 0 \\
\beta & \alpha & 0 \\
0 & 0 & \gamma
\end{array}\right]
$$

Note that the three columns of the matrix $T$, which will be denoted as $T_{a}, T_{b}$, and $T_{c}$, are linearly independent vectors and hence constitute a basis for $\mathbb{R}^{3}$ which will be denoted as $\mathcal{B}^{\prime}$. Without loss of generality, from this point onwards we will assume that $x=\left[x_{1}, x_{2}, x_{3}\right]^{\top} \in \mathbb{R}^{3}$ is a coordinate vector relative to the basis $\mathcal{B}^{\prime}$.

For the system $\dot{x}=A_{1} x$, an initial condition in the subspace defined by $V_{u}:=\operatorname{span}\left\{T_{a}, T_{b}\right\}$ will only excite the complex conjugate mode. Hence, a necessary condition for $(3)-(4)$ to have a limit cycle is that the subspace $V_{u}$ is not parallel to the switching surface $S$. Otherwise, any trajectory of the system $\dot{x}=A_{1} x$ starting in $S_{-}$would asymptotically approach the subspace $V_{u}$ and spiral away from the origin without reaching neither $S$ nor $\underline{S}$.

Similarly, any trajectory with an initial condition in the subspace $V_{s}:=T_{c}$ only excites the stable real mode. If $V_{s}$ is not parallel to the switching surface $S$ (i.e. $T_{c}$ is not orthogonal to the vector $C^{\top}$ ), then there exists a point $\bar{x} \in S_{-} \cap V_{s}$ such that the trajectory of (3) - (4) starting in $\bar{x}$ will converge asymptotically towards the origin. Due to the continuity of solutions in terms of the initial states [Khalil, 2000, Theorem 3.5], a simple argument shows that for points in $S_{-}$"arbitrarily close" to $S_{-} \cap V_{s}$, the associated switching times become arbitrarily large. This fact, as we will see in the following section, plays a very important role when analysing the stability of the system's limit cycle.

\section{MAIN RESULTS}

This section starts by providing a sufficient condition which guarantees that the system (3) - (4) possesses an asymptotically stable limit cycle in $\mathbb{R}^{3} \backslash \mathcal{M}_{s}$. The following subsections show how to overcome certain technicalities which might arise when one verifies the aforementioned condition.

\subsection{Stability analysis of the limit cycle}

A sufficient condition for the system (3) - (4) to have a globally asymptotically stable limit cycle in $\mathbb{R}^{3} \backslash \mathcal{M}_{s}$ is given by an adaptation of the results presented in [Gonçalves, 2000, Section 7.3] to the class of systems considered herein. These results are based on the discovery that impact maps can be represented as linear transformations parametrised by the switching times. The stability of the limit cycle is then ensured by proving that the impact maps are contractive around the points in $S_{+}$and $S_{-}$ which belong to the limit cycle. These points are denoted as $x_{0}^{\circ}$ and $x_{1}^{\circ}$, respectively.

There are three important aspects to consider during the aforementioned adaptation. The first one concerns the selection of the points $x_{0}^{*}$ and $x_{1}^{*}$ such that [Gonçalves, 2000, Proposition 7.1] holds. Given that $A$ is Hurwitz, let $P_{A}>0$ satisfy $P_{A} A+A^{\top} P_{A}<0$ and define the point $x_{0}^{*} \in S_{-}$as follows:

$$
x_{0}^{*}:=\left(d-C A^{-1} B \kappa\right) \frac{P_{A}^{-1} C^{\top}}{C P_{A}^{-1} C^{\top}}+A^{-1} B \kappa
$$

If $V_{s} \cap S_{-} \neq \varnothing$, let $x_{1}^{*}:=\bar{x}$; otherwise, $x_{1}^{*}:=x_{1}^{\circ}$. Our choice of $x_{0}^{*}$ guarantees that $C x_{0}^{*}(t) \neq d$ for all $t>0$, as shown in [Gonçalves, 2000, Section 7.2]. In a similar fashion, if $V_{s} \cap S_{-} \neq \varnothing$, then $C x_{1}^{*}(t) \neq d$ and $C x_{1}^{*}(t) \neq-d$ for all $t>0$. Otherwise, the functions $H_{2 a}(t)$ and $H_{2 b}(t)$ defined in [Gonçalves, 2000, Proposition 7.1] can be defined via continuation for all $t>0$ such that $C x_{1}^{*}(t)=-d$.

In the second place, the Lyapunov function $V_{1}\left(V_{2}\right)$ introduced in [Gonçalves, 2000, Section 7.3] has to be centred around the point in $S_{+}\left(S_{-}\right)$which belongs to the limit cycle. To do so, let $P_{i} \in \mathbb{R}^{2 \times 2}$ be symmetric, positive definite matrices, $g_{i} \in \mathbb{R}^{2}, \delta \in \mathbb{R}^{2}$ and $\alpha_{i} \in \mathbb{R}$ such that

$$
V_{i}(\delta)=\delta^{\top} P_{i} \delta-2 \delta^{\top} g_{i}+\alpha_{i}
$$

for $i=1,2$. Let $\Pi \in C^{\perp}\left(C^{\perp}\right.$ stands for the orthogonal complement of $C)$. Defining $d_{0}=\Pi^{\top} x_{0}^{\circ}-\Pi^{\top} x_{0}^{*}\left(d_{1}=\right.$ $\left.\Pi^{\top} x_{1}^{\circ}-\Pi^{\top} x_{1}^{*}\right)$ and fixing $g_{1}=P_{1} d_{0}\left(g_{2}=P_{2} d_{1}\right)$ and $\alpha_{1}=d_{0}^{\top} P_{1} d_{0}\left(\alpha_{2}=d_{1}^{\top} P_{2} d_{1}\right)$ centres the ellipse $V_{1}(\delta)=\varsigma$ $\left(V_{2}(\delta)=\varsigma\right)$ around $x_{0}^{\circ}\left(x_{1}^{\circ}\right)$.

In the third place, the conditions presented in [Gonçalves, 2000, Section 6.7.2] must also be modified to incorporate our particular choice of $g_{i}$ and $\alpha_{i}$ for $i=1,2$. This is done using standard algebra and is left to the reader.

The numerical verification of the conditions sketched herein is straightforward when lower and upper bounds on the set of expected switching times can be found. This is done by obtaining a finite sequence $t_{0}<t_{1}<\cdots<t_{k}$ 
whose elements belong to the set of expected switching times and verifying the conditions presented in [Gonçalves, 2000, Section 7.3] on $t=\left\{t_{i}\right\}, i=0,1, \ldots, k$. For large enough $k$, it can be shown that such conditions are also satisfied for all the set of expected switching times, as the latter reference explains.

Nevertheless, it was seen in Section 2.2 that one cannot always find an upper bound for all the sets of expected switching times associated with the class of systems under consideration. A methodology to address this situation is presented next.

3.2 Verifying the conditions for stability of the limit cycle. From this point onwards, it will be assumed that the set $V_{s} \cap S_{-}$is not empty. Define $\mathcal{T}_{2 a}\left(\mathcal{T}_{2 b}\right)$ as the set of expected switching times for the impact map $2 a(2 b)$ which takes points from $S_{-} \subset S\left(S_{-} \subset S\right)$ and maps them in $S_{+} \subset S\left(\underline{S}_{+} \subset \underline{S}\right)$. As discussed in section 2 , the sets $\mathcal{T}_{2 a}$ and $\mathcal{T}_{2 b}$ are not bounded, so a numerical verification of the conditions mentioned in Section 3.1 is futile. A methodology to overcome this problem is presented herein.

1.- Enforcing boundedness of $\mathcal{T}_{2 a}$ and $\mathcal{T}_{2 b}$. We start by characterising a neighbourhood $E_{\varepsilon}$ of points in $S$ centred around $V_{s} \cap S_{-}$. We also provide an estimation for a bounded set $I_{l}$ that any trajectory of (3) - (4) will eventually enter. If contraction of the impact maps around the limit cycle is guaranteed for points in this bounded set, one can simply wait until the considered trajectory of the system gets there; from that moment onwards, the trajectory will converge to the limit cycle. Using geometrical arguments, we provide an upper bound for the switching time of points in $S_{-}$which belong to $I_{l}$ but do not belong to $E_{\varepsilon}$. The impact maps for all such points can then shown to be contractive using the conditions mentioned in Section 3.1. Verifying the contraction of the impact maps for points which belong to $E_{\varepsilon}$ is deferred to the following subsection.

Start by fixing $\varepsilon>0$. The set

$$
E_{\varepsilon}:=\left\{x \in S \mid x_{1}^{2}+x_{2}^{2} \leq \varepsilon^{2}\right\}
$$

defines an $\varepsilon$-neighbourhood of the subspace $V_{s}$ in the switching surface $S$. For reasons that will become transparent later, the fixed value of $\varepsilon$ must be such that $E_{\varepsilon} \cap$ $V_{u}=\varnothing$ (see Theorem 3), $E_{\varepsilon} \cap\left\{x \in S \mid C A_{1} x=0\right\}=\varnothing$ (see Lemma 4 ), and $x_{1}^{\circ} \notin E_{\varepsilon}$ (see the remark after Theorem 7).

Now let $\bar{x}$ be the point which belongs to both $V_{s}$ and $S_{-}$. This point fulfills $C \bar{x}=d$ and $\bar{x}=\left[0,0, \overline{x_{3}}\right]^{\top}$ for a given $\overline{x_{3}}>0$. The following lemma shows how to coalesce the definitions of $E_{\varepsilon}$ and $\bar{x}$.

Lemma 1. Let $0_{i \times j}$ be an $i \times j$ matrix of zeros and let $I_{k}$ be the $k \times k$ identity matrix. An equivalent description for the set $E_{\varepsilon}$ is given by

where

$$
E_{\varepsilon}:=\left\{x_{1}^{*}+\Pi \delta_{1} \mid \quad \delta_{1}^{\top} P_{E} \delta_{1} \leq 1\right\}
$$

is positive definite.

$$
P_{E}:=\frac{1}{\varepsilon^{2}} \Pi^{\top}\left[\begin{array}{cc}
I_{2} & 0_{2 \times 1} \\
01 \times 2 & 0
\end{array}\right] \Pi
$$

Proof: The proof follows from straightforward algebra, the parametrisation of points in $S$ as $x_{1}^{*}+\Pi \delta_{1}$ where $x_{1}^{*}=\bar{x}$, and the fact that the coordinates $x_{1}$ and $x_{2}$ relative to the basis $\mathcal{B}^{\prime}$ are both zero for $\bar{x}$. The positive definiteness of $P_{E}$ is a direct consequence of $C T_{c} \neq 0$.

Since the matrix $A$ is Hurwitz and $|u| \leq \kappa$ is a bounded input, there is a bounded set $I_{l}$ that any trajectory of (3) - (4) will eventually enter, as the following proposition demonstrates.

Proposition 2. Recall the definition of $\Pi$ as a matrix belonging to the orthogonal complement of $C$. Let $\Pi_{1}$

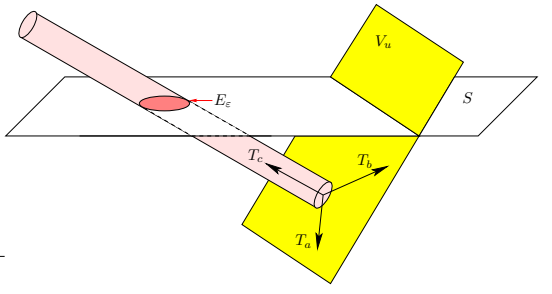

Fig. 2. The value of $\varepsilon>0$ defines the set $E_{\varepsilon}$.

and $\Pi_{2}$ be the columns of the matrix $\Pi$. Let $m \geq 2$ be an integer and pick $\psi_{i}$ for $i \in\{1, \ldots, m\}$ such that $0 \leq \psi_{1}<\ldots<\psi_{m}<\pi$. Define the row vector $F_{i}:=$ $\cos \left(\psi_{i}\right) \Pi_{1}^{\top}+\sin \left(\psi_{i}\right) \Pi_{2}^{\top}$. For the system (3) - (4),

$$
I_{l}:=\left\{x \mid \begin{array}{c}
\left|F_{1} x\right| \leq \kappa\left\|F_{1} e^{A t} B\right\|_{\mathcal{L}_{1}} \\
\vdots \\
\left|F_{m} x\right| \leq \kappa\left\|F_{m} e^{A t} B\right\|_{\mathcal{L}_{1}}
\end{array}\right\}
$$

defines a bounded set that any trajectory will eventually enter.

Proof: See [Gonçalves, 2000, Proposition 7.2].

The definitions introduced below will be needed to show that the switching times for all points in $S$ which belong to $I_{l}$ but do not belong to $E_{\varepsilon}$ are bounded. Let $x_{3_{p}}$ be a fixed nonzero real number between 0 and $\overline{x_{3}}$. Consider a plane $P$ parallel to the subspace $V_{u}$ as follows:

$$
P:=\left\{x \mid[0,0,1] x=x_{3_{p}}\right\}
$$

For $\varrho>0$, define a circle in $V_{u}$ relative to the basis $\mathcal{B}^{\prime}$ by $\mathcal{C}_{1}(\varrho)=\left\{x \in V_{u} \mid x_{1}^{2}+x_{2}^{2}=\varrho^{2}\right\}$. A circle in $P$ relative to the basis $\mathcal{B}^{\prime}$ can be defined analogously by $\mathcal{C}_{2}(\varrho)=\left\{x \in P \mid x_{1}^{2}+x_{2}^{2}=\varrho^{2}\right\}$. Let $\varrho_{1}\left(\varrho_{2} ; \underline{\varrho_{2}}\right)$ be the minimum value of $\varrho$ such that $\mathcal{C}_{1}(\varrho) \cap S \neq \varnothing\left(\mathcal{C}_{2}(\varrho) \cap S \neq \varnothing\right.$; $\left.\mathcal{C}_{2}(\varrho) \cap \underline{S} \neq \varnothing\right)$ (see Figure 3$)$. Now define $x_{3 \max }$ as the maximum distance between a point in $S \cap I_{l}$ and the subspace $V_{u}$.

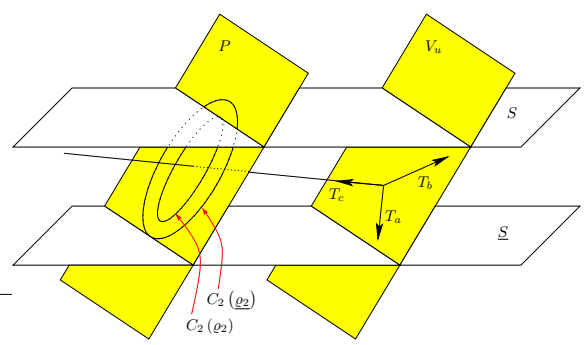

Fig. 3. Planes $V_{u}$ and $P$; circles $C_{2}\left(\varrho_{2}\right)$ and $C_{2}\left(\underline{\varrho_{2}}\right)$

As the following theorem demonstrates, defining the sets $P$ and $E_{\varepsilon}$ permits the computation of an upper bound for the switching time of all points in the switching surface $S_{-}$which belong to the set $I_{l}$ but do not belong to the neighbourhood $E_{\varepsilon}$.

Theorem 3. Fix $x_{3 p} \neq 0$ between 0 and $\overline{x_{3}}$. Pick a value of $\varepsilon$ such that $\varrho_{2} \geq \varepsilon>0$. Define the time instants

$$
\begin{aligned}
t_{1} & :=\left(\ln \varrho_{1}-\ln \varepsilon\right) / \alpha, & t_{2} & :=2 \pi / \beta, \\
t_{3} & :=\left(\ln x_{3 p}-\ln x_{3 \max }\right) / \gamma, & \breve{t} & :=\max \left(t_{1}, t_{3}\right)+t_{2}, \\
t_{1}^{\prime} & :=\left(\ln \varrho_{2}-\ln \varepsilon\right) / \alpha, & t_{2}^{\prime} & :=\pi / \beta, \\
t_{3}^{\prime} & :=\left(\ln x_{3 p}-\ln x_{3 \max }\right) / \gamma, & \breve{t} & :=\max \left(t_{1}^{\prime}, t_{3}^{\prime}\right)+t_{2}^{\prime}
\end{aligned}
$$

for the system

$$
\dot{x}=A_{1} x, \quad x(0) \in\left(S \cap I_{\text {inv }}\right) \backslash E_{\varepsilon}
$$

Then there exists at least one time instant $t_{s}\left(\breve{t}_{s}\right)$ which fulfills $x\left(t_{s}\right) \in S\left(x\left(\breve{t}_{s}\right) \in \underline{S}\right)$ and $0<t_{s} \leq \breve{t}\left(0<\breve{t}_{s} \leq \breve{t}\right)$. 
Proof: Define $r:=\sqrt{x_{1}^{2}+x_{2}^{2}}$ and $\theta:=\arctan \left(x_{2} / x_{1}\right)$. Hence, $\dot{x}=A_{1} x$ can be expressed via the uncoupled differential equations $\dot{r}=\alpha r, \dot{\theta}=\beta$, and $\dot{w}_{3}=\gamma w_{3}$ with initial conditions $r(0) \geq \varepsilon, \theta(0)=\theta_{0}$, and $w_{3}(0) \leq$ $w_{3 \max }$. By assumption, $\alpha>0, \beta>0$, and $\gamma<0$, so straightforward calculations show that $r(\breve{t})>r\left(t_{1}\right) \geq \varrho_{1}$, that $w_{3 p} \geq w_{3}\left(t_{3}\right)>w_{3}(\breve{t})>0$, and that $\theta(\breve{t})=\theta_{0}+$ $\beta \max \left(t_{1}, t_{3}\right)+2 \pi$. From the second set of inequalities it can be seen that the trajectory of (8) remains between the planes $P$ and $V_{u}$ for all time $t \geq t_{3}$. In addition, after the time $t \geq \max \left(t_{1}, t_{3}\right)$ has elapsed, the distance between the subspace $V_{s}$ and the trajectory of (8) has grown to be greater than or equal to $\varrho_{1}$ (this is given by the first set of inequalities). Finally, the expression for $\theta(\breve{t})$ shows that the additional time interval $t_{2}$ allows a complete revolution around $V_{s}$ to occur, thus completing the proof. As an aside, observe that the choice of $\varepsilon$ guarantees that $E_{\varepsilon} \cap V_{u}=\varnothing$.

The proof for $\breve{t}_{s}$ is similar and is thus left to the reader. Notice that in this case only half a revolution around $V_{s}$ is needed; this is a direct consequence of the fact that $\varrho_{2}>\varrho_{1}>\varrho_{2}$.

The above theorem permits the conditions mentioned in Section 3.1 to be verified for all points in $S \cap I_{l}$ which do not belong to $E_{\varepsilon}$. As the points in $E_{\varepsilon}$ cannot be neglected, the next section will show how to deal with them.

2.- Investigation of the set $E_{\varepsilon}$. This subsection shows how to verify that trajectories starting in the set $E_{\varepsilon} \backslash V_{s}$ converge to the limit cycle of system (3) - (4). Although the approach used here involves showing that the impact maps are contracting around the limit cycle, its novelty resides in the fact that it does not recur to the switching times associated with points in $E_{\varepsilon} \backslash V_{s}$.

We start by proving that, under some assumptions, a trajectory of $\dot{x}=A_{1} x$ which starts in a point belonging to $E_{\varepsilon} \backslash V_{s}$ will take at least some time $t_{E, 2 a}\left(t_{E, 2 b}\right)$ to reach the switching surface $S(\underline{S})$.

Lemma 4. Define $w_{2 a}(t):=\frac{C e^{A_{1} t} \Pi}{d-C x_{1}^{*}(t)}$ for $t>0$ and $w_{2 a}(0):=\frac{-C A_{1} \Pi}{C A_{1} x_{1}^{*}}$. Let

$\tilde{d}_{2 a}(t)=\frac{1}{2} P_{E}{ }^{-1} w_{2 a}^{\top}(t), \quad d_{2 a}(t)=\frac{\tilde{d}_{2 a}(t)}{\left(\tilde{d}_{2 a}^{\top}(t) P_{E} \tilde{d}_{2 a}(t)\right)^{1 / 2}}$

for all $t>0$. Assume that the value of $\varepsilon$ chosen to characterise the set $E_{\varepsilon}$ is such that

$$
\left(w_{2 a}(0) d_{2 a}(0)-1\right)\left(-w_{2 a}(0) d_{2 a}(0)-1\right)>0
$$

Let $t_{E, 2 a}$ be the smallest value of $t \in \mathcal{T}_{2 a} \backslash\{0\}$ for which the expression

$$
\left(w_{2 a}(t) d_{2 a}(t)-1\right)\left(-w_{2 a}(t) d_{2 a}(t)-1\right)
$$

is less than or equal to zero. Then, all points in $E_{\varepsilon}$ $V_{s}$ which switch in $S$ have an associated switching time greater than or equal to $t_{E, 2 a}$.

It can also be shown that all points in $E_{\varepsilon} \backslash V_{s}$ which switch in $\underline{S}$ have an associated switching time greater than or equal to $t_{E, 2 b}$ by defining $w_{2 b}(t)=\frac{C e^{A_{1} t} \Pi}{-d-C x_{1}^{*}(t)}$ for $t \in \mathcal{T}_{2 b}$ and using this expression instead of $w_{2 a}(t)$ in the abovementioned result.

Proof: Define $S_{t_{2 a}}\left(S_{t_{2 b}}\right)$ as the set of initial conditions $x_{1_{a}} \in S_{-}\left(x_{1_{b}} \in S_{-}\right)$such that $-d \leq C x(t) \leq d$ on $\left[0, t_{2 a}\right]$ and $C x\left(t_{2 a}\right)=d\left(-d \leq C x(t) \leq d\right.$ on $\left[0, t_{2 b}\right]$ and $\left.C x\left(t_{2 b}\right)=-d\right)$. Corollary 4.1 in Gonçalves [2000] shows that, for all $t_{2 a}$ in $\mathcal{T}_{2 a} \backslash\{0\}$, the set $S_{t_{2 a}}$ is a subset of the linear manifold $\mathbf{S}_{t_{2 a}}:=\left\{x_{1}^{*}+\Pi \delta_{1} \mid w_{2 a}\left(t_{2 a}\right) \delta_{1}=1\right\}$. For $t_{2 a}=0$, the set $\mathbf{S}_{t_{2 a}=0}$ is given by $\left\{x_{1}^{*}+\Pi \delta_{1} \mid w_{2 a, 0} \delta_{1}=1\right\}$ since $w_{2 a, 0} \delta_{1}=\lim _{t \rightarrow 0} w_{2 a}(t) \delta_{1}$. It is then obvious that if the set $E_{\varepsilon}$ has no points in common with $\mathbf{S}_{t_{2 a}}$ for all $t_{2 a} \in\left[0, t_{E, 2 a}\right)$, then the switching time for all points in $E_{\varepsilon}$ must be equal to or greater than $t_{E, 2 a}$.

As a shorthand, we will be using $w_{2 a t}$ for $w_{2 a}\left(t_{2 a}\right)$ and $d_{2 a t}$ for $d_{2 a}\left(t_{2 a}\right)$. Define the sets

$$
\begin{gathered}
\mathbf{S}_{E, t}^{\prime}:=\left\{\delta_{1} \in \mathbb{R}^{2} \mid \delta_{2 a t}^{\top} P_{E} \delta_{1}=1\right\} \\
\mathbf{S}_{w, t}^{\prime}:=\left\{\delta_{1} \in \mathbb{R}^{2} \mid w_{2 a t} \delta_{1}=1\right\}
\end{gathered}
$$

Straightforward manipulations show that $\delta_{2 a t}^{\top} P_{E}=c w_{2 a t}$ for $c=\left(w_{2 a t}^{\top} P_{E} w_{2 a t}\right)^{-1 / 2}$, so the sets $\mathbf{S}_{w, t}^{\prime}, \mathbf{S}_{E, t}^{\prime}$ and $-\mathbf{S}_{E, t}^{\prime}$ represent lines in $\mathbb{R}^{2}$ parallel to each other. Furthermore, $\mathbf{S}_{E, t}^{\prime}$ and $-\mathbf{S}_{E, t}^{\prime}$ are tangent to the ellipse $\delta_{1}^{\top} P_{E} \delta_{1}=$ 1 at the points $\delta_{1}=\delta_{2 a t}$ and $\delta_{1}=-\delta_{2 a t}$. It then follows that $\mathbf{S}_{t_{2 a}} \cap E_{\varepsilon} \neq \varnothing$ if and only if $\left(w_{2 a t} d_{2 a t}-1\right)\left(-w_{2 a t} d_{2 a t}-\right.$ $1)>0$. This equivalence is explained by the fact that $\left(w_{2 a t} d_{2 a t}-1\right)$ and $\left(w_{2 a t}\left(-d_{2 a t}\right)-1\right)$ have the same sign if and only if $\mathbf{S}_{w, t}^{\prime}$ is not located between the lines $\mathbf{S}_{E, t}^{\prime}$ and $-\mathbf{S}_{E, t}^{\prime}$. This means that no $\delta_{1}$ can satisfy $w_{2 a t} \delta_{1}=1$ and $\delta_{1}^{\top} P_{E} \delta_{1} \leq 1$ simultaneously, as Figure 4 shows. In this case, the sets $E_{\varepsilon}$ and $\mathbf{S}_{t_{2 a}}$ have no points in common, so our claim follows.

The proof for $t_{E, 2 b}$ follows the same line of reasoning and is thus omitted.

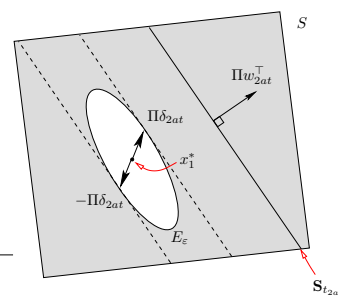

Fig. 4. $\quad \mathbf{S}_{t_{2 a}} \cap E_{\varepsilon} \neq \varnothing$. The dashed lines represent the sets $\left\{x_{1}^{*}+\Pi \delta_{1} \mid \delta_{1} \in \mathbf{S}_{E, t}^{\prime}\right\} \quad$ and $\left\{x_{1}^{*}+\Pi \delta_{1} \mid \delta_{1} \in-\mathbf{S}_{E, t}^{\prime}\right\}$.

Using time $t_{E, 2 a}\left(t_{E, 2 b}\right)$, it can be determined how close to the subspace $V_{u}$ the trajectory has to be when it reaches $S(\underline{S})$.

Lemma 5. Let $S_{d} \subset\left(S_{-} \backslash V_{s}\right)\left(S_{-d} \subset\left(S_{-} \backslash V_{s}\right)\right)$ be the set of points that will eventually switch in $S(\underline{S})$. Define $t_{E, 2 a}$ and $t_{E, 2 b}$ as in Lemma 4 . Let $x_{3_{E+}}$ be the maximum distance between a point in $E_{\varepsilon}$ and the subspace $V_{u}$. Define $x_{3_{E 2 a}}:=x_{3_{E+}} \exp \left(\gamma t_{E, 2 a}\right)$ and $x_{3_{E 2 b}}:=x_{3_{E+}} \exp \left(\gamma t_{E, 2 b}\right)$. Then any trajectory of $\dot{x}=A_{1} x$ starting in $S_{d} \cap E_{\varepsilon}$ $\left(S_{-d} \cap E_{\varepsilon}\right)$ will not be away from the subspace $V_{u}$ by a distance greater than $x_{3_{E 2 a}}\left(x_{3_{E 2 b}}\right)$ when it switches at $S(\underline{S})$.

Proof: The proof follows from the definition of $t_{E, 2 a}, t_{E, 2 b}$ and $x_{3 E+}$ by recalling that the stable behaviour of $\dot{x}=A_{1} x$ is uncoupled from the unstable one.

An additional geometrical argument then characterises subsets of $S$ and $\underline{S}$ that this trajectory will reach.

Lemma 6 . Assume that the value of $\varepsilon$ used to characterise the set $E_{\varepsilon}$ complies with the conditions given in Theorem 3 and Lemma 4. Define

$$
\varrho_{E 2 a}:=\frac{d+\tilde{c_{3}} x_{3_{E 2 a}}}{\sqrt{\left(\tilde{c_{1}}\right)^{2}+\left(\tilde{c_{2}}\right)^{2}}}, \quad q:=\varrho_{E 2 a} \exp \left(\frac{2 \pi \alpha}{\beta}\right)
$$

where $\left[\begin{array}{ccc}\tilde{c_{1}} & \tilde{c_{2}} & \tilde{c_{3}}\end{array}\right]:=C$, and $x_{3_{E 2 a}}$ is defined as in Lemma 5. Let the set $S_{\eta, 2 a}$ be defined by 


$$
\left\{C A_{1} x \mid C x=d, x_{1}^{2}+x_{2}^{2} \leq q^{2}, 0 \leq x_{3} \leq x_{3_{E 2 a}}\right\}
$$

and denote its infimum and supremum values as $\eta_{a 1}$ and $\eta_{a 2}$, respectively. If $\eta_{a 1}$ is negative, then re-define it as $\eta_{a 1}:=0$. Then all trajectories of $\dot{x}=A_{1} x$ which start in the set $E_{\varepsilon} \backslash V_{s}$ and eventually switch in $S$ will do so in the set $S_{E 2 a} \subset S$ defined by

$$
S_{E 2 a}:=\left\{x \in S \mid 0 \leq x_{3} \leq x_{3_{E 2 a} a}, \eta_{a 1} \leq C A_{1} x \leq \eta_{a 2}\right\}
$$

Now define $q_{2 b}$ via the substitution of $x_{3_{E 2 a}}$ by $x_{3_{E 2 b}}$ in (10). Define the set $S_{\eta, 2 b}$ by

$$
\left\{C A_{1} x \mid C x=-d, x_{1}^{2}+x_{2}^{2} \leq q_{2 b}^{2}, 0 \leq x_{3} \leq x_{3_{E 2 b}}\right\}
$$

and denote its infimum and supremum values by $\eta_{b 1}$ and $\eta_{b 2}$, respectively. If $\eta_{b 2}$ is positive, then re-define it as $\eta_{b 2}:=0$. Then all trajectories of $\dot{x}=A_{1} x$ which start in the set $E_{\varepsilon} \backslash V_{s}$ and eventually switch in $\underline{S}$ will do so in the set $S_{E 2 b} \subset \underline{S}$ defined by

$$
\left\{x \in \underline{S} \mid 0 \leq x_{3} \leq x_{3_{E 2 b}}, \eta_{b 1} \leq C A_{1} x \leq \eta_{b 2}\right\}
$$

Proof: We begin by noticing that $x_{3_{E 2 a}}$ can be used, as in Section 3.2.1, to define a plane $P_{E 2 a}$ which is parallel to the subspace $V_{u}$. The value of $\varrho_{2}$ associated with $P_{E 2 a}$ is denoted as $\varrho_{E 2 a}$ and is greater than $\varepsilon$ due to the assumptions placed on the latter. Now recall the definitions $r:=\sqrt{x_{1}^{2}+x_{2}^{2}}, \theta:=\arctan \left(x_{2} / x_{1}\right)$, and the fact that the solutions at time $t=2 \pi / \beta$ of the differential equations $\dot{r}=\alpha r ; \dot{\theta}=\beta$ with initial conditions $r(0)=\varrho_{E 2 a}$; $\theta(0)=\theta_{0}$ are equal to $q ; \theta_{0}+2 \pi$. This shows that once any trajectory of $\dot{x}=A_{1} x$ starting in $S_{d} \cap E_{\varepsilon}$ has reached the set $\left\{x \in \mathbb{R}^{3} \mid x_{1}^{2}+x_{2}^{2}=\varrho_{E 2 a}^{2}\right\}$, it must undergo another complete revolution around $V_{s}$ before reaching $\left\{x \in \mathbb{R}^{3} \mid x_{1}^{2}+x_{2}^{2} \geq q^{2}\right\}$. Given the geometrical interpretation of $\varrho_{E 2 a}$, this cannot be done without reaching the switching surfaces $S$ and $\underline{S}$ first.

To prove that a point in $S_{d} \cap E_{\varepsilon}$ cannot eventually switch outside $S_{E 2 a}$, we proceed by contradiction. Suppose that a point $\tilde{x} \in S_{d} \cap E_{\varepsilon}$ switches outside $S_{E 2 a}$. Notice that switching in the set $\left\{x \in S \mid x_{3}<0\right\}$ is impossible due to the dynamics of the system. Furthermore, Lemma 5 shows that switching in the set $\left\{x \in S \mid x_{3_{E 2 a}}<x_{3}\right\}$ is impossible too. Hence, the point in $S_{d} \cap E_{\varepsilon}$ is restricted to switch in the set $S_{\times}:=S_{\times_{1}} \cup S_{\times_{2}}$ where

$$
\begin{aligned}
& S_{\times_{1}}:=\left\{x \in S \mid 0 \leq x_{3} \leq x_{3_{E 2 a}}, \eta_{1}>C A_{1} x\right\} \\
& S_{\times_{2}}:=\left\{x \in S \mid 0 \leq x_{3} \leq x_{3_{E 2 a}}, \eta_{2}<C A_{1} x\right\}
\end{aligned}
$$

Since the definitions of $\eta_{1}$ and $\eta_{2}$ ensure that the set

$$
\left\{x \in \mathbb{R}^{3} \mid C x=d, \quad 0 \leq x_{3} \leq x_{3_{E 2 a}}, \quad x_{1}^{2}+x_{2}^{2} \leq q^{2}\right\}
$$

is contained in the set $S_{E 2 a}$, it is readily seen that $x_{1}^{2}+x_{2}^{2}>q^{2}$ for all points $x \in S_{\times}$. This contradicts the information presented in the previous paragraph. In addition, all trajectories of $\dot{x}=A_{1} x$ starting in $S_{-}$are restricted to switch either in the set $S_{+}$or in the set $\underline{S}_{+}$. Hence, our claim follows.

The proof for $S_{E 2 b}$ follows the same line of reasoning and is thus omitted.

We now present a condition which guarantees that the mapping of points in $E_{\varepsilon} \backslash V_{s}$ to the aforementioned subsets of $S$ and $\underline{S}$ is contracting around the limit cycle.

Define $S_{E S}:=S_{E 2 a}$ and $S_{E S}:=-S_{E 2 b}$. We furthermore consider the following notation: if $S$ is a set, then define the set $S-x_{s}$ as

$$
S-x_{s}:=\left\{x-x_{s} \mid x \in S\right\}
$$

To verify that trajectories starting in the set $E_{\varepsilon} \backslash V_{s}$ converge to the limit cycle of system (3) - (4), it is sufficient to show that the impact maps which take points from
$E_{\varepsilon} \backslash V_{s}$ and map them in $S$ and $\underline{S}$ are contracting. A sufficient condition which guarantees such contraction is

$$
\begin{array}{r}
\left\{\begin{array}{ll}
\max & V_{1}\left(\delta_{2 a}\right) \\
\text { s.t. } \Pi \delta_{2 a} \in S_{E S}-x_{0}^{*}
\end{array}\right\}<\left\{\begin{array}{l}
\min V_{2}\left(\delta_{1}\right) \\
\text { s.t. } \Pi \delta_{1} \in E_{\varepsilon}-x_{1}^{*}
\end{array}\right\} \\
\left\{\begin{array}{ll}
\max & V_{1}\left(\delta_{2 b}\right) \\
\text { s.t. } \Pi \delta_{2 b} \in S_{E \underline{S}}-x_{0}^{*}
\end{array}\right\}<\left\{\begin{array}{ll}
\min V_{2}\left(\delta_{1}\right) \\
\text { s.t. } \Pi \delta_{1} \in E_{\varepsilon}-x_{1}^{*}
\end{array}\right\}
\end{array}
$$

We now present a set of linear matrix inequalities which, if fulfilled, guarantee that conditions (11) hold.

Theorem $\%$. Define $\Gamma:=[0,0,1]$ and let $M$ be a $3 \times 3$ matrix with rows given by $\Gamma, C$, and $C A_{1}$, respectively. Let

$$
\begin{array}{ll}
\vartheta_{a, 1}:=\left[0, d, \eta_{a 1}\right]^{\top} & \vartheta_{a, 2}:=\left[x_{3_{E 2 a}}, d, \eta_{a 1}\right]^{\top} \\
\vartheta_{a, 3}:=\left[0, d, \eta_{a 2}\right]^{\top} & \vartheta_{a, 4}:=\left[x_{3_{E 2 a}}, d, \eta_{a 2}\right]^{\top} \\
\vartheta_{b, 1}:=\left[0, d,-\eta_{b 1}\right]^{\top} & \vartheta_{b, 2}:=\left[-x_{3_{E 2 b}}, d,-\eta_{b 1}\right]^{\top} \\
\vartheta_{b, 3}:=\left[0, d,-\eta_{b 2}\right]^{\top} & \vartheta_{b, 4}:=\left[-x_{3_{E 2 b}}, d,-\eta_{b 2}\right]^{\top}
\end{array}
$$

where $w_{3_{E 2 a}}, w_{3_{E 2 b}}, \eta_{a 1}, \eta_{a 2}, \eta_{b 1}$, and $\eta_{b 2}$ define the sets $S_{E S}$ and $S_{E \underline{S}}$.

Define $\sigma_{a, i}=V_{1}\left(\Pi^{\top}\left\{M^{-1} \vartheta_{a, i}-x_{0}^{*}\right\}\right)$ and $\sigma_{b, i}=$ $V_{1}\left(\Pi^{\top}\left\{M^{-1} \vartheta_{b, i}-x_{0}^{*}\right\}\right)$ for $i \in\{1,2,3,4\}$. If there exist nonnegative scalars $\tau_{a, i}$ and $\tau_{b, i}$ such that the matrices

$$
\begin{aligned}
& {\left[\begin{array}{cc}
P_{2}+\tau_{a, i} P_{E} & -g_{2} \\
-g_{2}^{\top} & \alpha_{2}-\sigma_{a, i}-\tau_{a, i}
\end{array}\right]} \\
& {\left[\begin{array}{cc}
P_{2}+\tau_{b, i} P_{E} & -g_{2} \\
-g_{2}^{\dagger} & \alpha_{2}-\sigma_{b, i}-\tau_{b, i}
\end{array}\right]}
\end{aligned}
$$

are positive definite for all $i \in\{1,2,3,4\}$, then conditions (11) hold.

Proof: The matrix $M$ can be shown to be non-singular by considering that $A_{1} \neq \xi I_{3}$ together with the fact that the vector $\Gamma^{\top} \notin V_{u}$.

Let $i \in\{1,2,3,4\}$. The fact that $M^{-1}$ exists shows that $S_{E S}\left(S_{E \underline{S}}\right)$ can be visualised as the set of points in $S$ enclosed within the parallelogram with vertices $M^{-1} \vartheta_{a, i}$ $\left(M^{-1} \vartheta_{b, i}\right)$. Since the function $V_{1}$ is convex by construction (see section 3.1), maximising its value over the sets $S_{E S}-$ $x_{0}^{*}$ and $S_{E S}-x_{0}^{*}$ can be done by computing its value at the points $\bar{\Pi}^{\top}\left\{M^{-1} \vartheta_{a, i}-x_{0}^{*}\right\}$ and $\Pi^{\top}\left\{M^{-1} \vartheta_{b, i}-x_{0}^{*}\right\}$; the biggest of such values is the desired result. Hence, enforcing conditions (11) is equivalent to asking the inequalities

$$
\begin{aligned}
& \sigma_{a, i}<V_{2}(\delta)=\delta^{\top} P_{2} \delta-2 \delta^{\top} g_{2}+\alpha_{2} \\
& \sigma_{b, i}<V_{2}(\delta)=\delta^{\top} P_{2} \delta-2 \delta^{\top} g_{2}+\alpha_{2}
\end{aligned}
$$

to hold for all $\delta \neq 0$ such that $\delta^{\top} P_{E} \delta \leq 1$. It is obvious that if there exist nonnegative scalars $\tau_{a, 1}$ and $\tau_{b, 1}$ such that

$$
\begin{aligned}
& \delta^{\top} P_{2} \delta-2 \delta^{\top} g_{2}+\alpha_{2}-\sigma_{a, i}-\tau_{a, i}\left(1-\delta^{\top} P_{E} \delta\right)>0 \\
& \delta^{\top} P_{2} \delta-2 \delta^{\top} g_{2}+\alpha_{2}-\sigma_{b, i}-\tau_{b, i}\left(1-\delta^{\top} P_{E} \delta\right)>0
\end{aligned}
$$

hold, then the aforementioned inequalities hold too. Realising that one can write this as

$$
\begin{aligned}
& {\left[\begin{array}{l}
\delta \\
1
\end{array}\right]^{\top}\left[\begin{array}{cc}
P_{2}+\tau_{a, i} P_{E} & -g_{2} \\
-g_{2}^{\top} & \alpha_{2}-\sigma_{a, i}-\tau_{a, i}
\end{array}\right]\left[\begin{array}{l}
\delta \\
1
\end{array}\right]>0} \\
& {\left[\begin{array}{l}
\delta \\
1
\end{array}\right]^{\top}\left[\begin{array}{cc}
P_{2}+\tau_{b, i} P_{E} & -g_{2} \\
-g_{2}^{\top} & \alpha_{2}-\sigma_{b, i}-\tau_{b, i}
\end{array}\right]\left[\begin{array}{l}
\delta \\
1
\end{array}\right]>0}
\end{aligned}
$$

completes the proof.

Remark: For Theorem 7 to be useful, the set $E_{\varepsilon}$ must be defined in such a way that it does not contain the point $x_{1}^{\circ}$. If it did,

$$
\begin{aligned}
& \min V_{2}\left(\delta_{1}\right) \\
& \text { s.t. } \Pi \delta_{1} \in E_{\varepsilon}-x_{1}^{*}
\end{aligned}
$$


would be equal to zero and, given that $V_{1}(\delta)$ is nonnegative for all values of $\delta$, the inequalities in (11) would never be fulfilled.

\section{EXAMPLE.}

The following example is a PWL version of the third order dimensionless Goodwin oscillator model whose parameters are congruent with those presented in Stan et al. [2007]. The SISO LTI block obeys

$$
\begin{aligned}
\dot{x} & =\left[\begin{array}{ccc}
-0.5 & 0 & 0 \\
0.5 & -0.5 & 0 \\
0 & 0.5 & -0.5
\end{array}\right] x+\left[\begin{array}{l}
1 \\
0 \\
0
\end{array}\right] u \\
y & =\left[\begin{array}{lll}
0 & 0 & 1
\end{array}\right] x
\end{aligned}
$$

while the saturation block is described by

$$
u(t)= \begin{cases}1 / 2 & \text { if } y(t)<-1 / 9 \\ -9 / 2 y(t) & \text { if }|y(t)| \leq 1 / 9 \\ -1 / 2 & \text { if } y(t)>1 / 9\end{cases}
$$

The feedback system has a locally stable limit cycle that switches four times per cycle with period $t^{\circ} \approx 2(1.5398+$ 2.0911). The intersection of the limit cycle with the switching surface $S$ occurs at $x_{0}^{\circ} \approx\left[\begin{array}{lll}0.0652, & 0.2634, & 0.1111\end{array}\right]^{\top}$ and $x_{1}^{\circ} \approx\left[\begin{array}{lll}-0.5067, & -0.0352, & 0.1111\end{array}\right]^{\top}$. The set $I_{l}$ was constructed according to Proposition 2 by selecting $m=$ 18 and $\psi_{i}=(m-1)(\pi / 18)$. The choice of $x_{3 p} \approx 0.0809$ and $\varepsilon=\varrho_{2} \approx 0.6111$ yields $\mathcal{T}_{2 a}=[0,15]$ and $\mathcal{T}_{2 b}=[0.47,18.43]$, (refer to section 3.2.1 and [Gonçalves, 2000, Proposition 7.5]). For this system, the Lyapunov functions on $S$ are

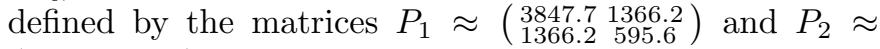

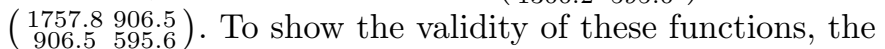
system was simulated on the time interval $[0,30]$ starting from the initial condition $x(0)=\left[\begin{array}{lll}0.6, & 0.6, & 1 / 9\end{array}\right]^{\top}$. The most relevant results of such simulation are shown in table 1; as expected, the value of the Lyapunov functions

\begin{tabular}{|c|c|c|c|c|}
\hline $\mathrm{t}$ & \multicolumn{3}{|c|}{$x(t)$} & $V_{i}$ \\
\hline 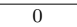 & 0.6, & $\overline{0.6,}$ & $\mid \overline{0.1111]}$ & $V_{1}=73.6639$ \\
\hline 3.4664 & -0.7172 & -0.2272 & $0.1111]$ & $V_{2}=17.9082$ \\
\hline 4.7706 & -0.3091 & -0.3949 , & $-0.1111]$ & $V_{1}=8.1239$ \\
\hline 7.2691 & 0.6247 & 0.1311, & $-0.1111]$ & $V_{2}=3.9507$ \\
\hline 8.895 & 0.193 & 0.329 & $0.1111]$ & $V_{1}=1.8294$ \\
\hline 10.9568 & -0.5745 & -0.0873, & $0.1111]$ & $V_{2}=1.1052$ \\
\hline 12.774 & -0.1357 & -0.2989 , & $-0.1111]$ & $V_{1}=0.5203$ \\
\hline 14.608 & 0.546 & 0.0654, & $-0.1111]$ & $V_{2}=0.3411$ \\
\hline 16.5382 & 0.1051 , & 0.2833 , & $0.1111]$ & $V_{1}=0.1618$ \\
\hline 18.2465 & -0.5296 & -0.052 & $0.1111]$ & $V_{2}=0.1102$ \\
\hline 20.2434 & -0.0881 & -0.2748 & $-0.1111]$ & $V_{1}=0.0524$ \\
\hline 21.8804 & 0.5201 & 0.0449 , & $-0.1111]$ & $V_{2}=0.0364$ \\
\hline 23.9165 & 0.0784 & 0.2700 & $0.1111]$ & $V_{1}=0.0173$ \\
\hline 25.5127 & -0.5145 & -0.0408 & 0.1111 & $V_{2}=0.0122$ \\
\hline 27.5716 & -0.0729 & -0.2672 , & $-0.1111]$ & $V_{1}=0.0058$ \\
\hline 29.1442 & 0.5113 & 0.0385, & $-0.1111]$ & $V_{2}=0.0041$ \\
\hline
\end{tabular}
decreases every time the trajectory reaches a switching surface.

Table 1. Results of the system's simulation over the time interval $[0,30]$ with initial condition $x(0)=\left[\begin{array}{lll}0.6, & 0.6,1 / 9\end{array}\right]^{\top}$. The first and second columns show the time instants in which the system's trajectory reaches a switching surface and the points in which this happens, while the third one gives the value of the appropriate Lyapunov function at such points.

\section{CONCLUSIONS.}

This paper presented a methodology to analyse the global stability of the limit cycle of a PWL saturation system which cannot be directly analysed using the methodology presented in Stan [2005]. It is based on the construction of quadratic Lyapunov functions on the system's switching surfaces and covers the case in which the expected switching times associated with the system's impact maps are arbitrarily large. The first step involves defining a subset of the system's switching surfaces in which the switching times are bounded; the methodology developed by Gonçalves [2000] is then applied to the study of such subset. The second step recurs to a geometrical argument in order to guarantee that all the trajectories which do not start in the aforementioned subset converge either to the origin or to the limit cycle. Taken together, both steps achieve the desired objective.

Future work involves the extension of the present results to higher-dimensional systems, thus opening the door to the analysis of models other than the third-order Goodwin oscillator presented herein.

\section{REFERENCES}

U. Boscain. Stability of planar switched systems: the linear single input case. SIAM Journal on Control and Optimization, 41(1):89-112, 2002.

M. S. Branicky. Multiple Lyapunov functions and other analysis tools for switched and hybrid systems. IEEE Transactions on Automatic Control, 43(4):475$482,1998$.

A. Goldbeter. Biochemical oscillations and cellular rhytms: the molecular bases of periodic and chaotic behaviour. Cambridge University Press, UK, 1996.

J. M. Gonçalves. Constructive global analysis of hybrid systems. PhD thesis, Massachusetts Institute of Technology, September 2000. URL http: //www-control.eng.cam.ac.uk/Homepage/ /papers/cued_control_893.pdf.

J. M. Gonçalves. Regions of stability for limit cycle oscillations in piecewise linear systems. IEEE Transactions on Automatic Control, 50(11):1877-1882, Nov. 2005.

R. A. Horn and C. R. Johnson. Matrix analysis. Cambridge University Press, UK, first edition, 1985.

Y. Iwatani and S. Hara. Stability tests and stabilization for piecewise linear systems based on poles and zeros of subsystems. Automatica, 42(10):1685-1695, 2006.

M. Johansson and A. Rantzer. Computation of piecewise quadratic Lyapunov functions for hybrid systems. IEEE Transactions on Automatic Control, 43(4):555559, April 1998.

H. Khalil. Nonlinear systems. Prentice Hall, USA, third edition, 2000.

M. Margaliot. Stability analysis of switched systems using variational principles: an introduction. Automatica, 42 (12):2059-2057, Dec. 2006.

E. Mosekilde. Topics in nonlinear dynamics; applications to physics, biology, and economic systems. World Scientific Press, Singapore, 1997.

S. Petterson and B. Lennartson. Hybrid system stability and robustness verification using linear matrix inequalities. International Journal of Control, 75:1335-1355, 2002.

S. Prajna and A. Papachristodoulou. Analysis of switched and hybrid systems - beyond piecewise quadratic methods. In American Control Conference (ACC), Denver, CO, USA, 2003.

G.-B. Stan. Global analysis and synthesis of oscillations: a dissipativity approach. $\mathrm{PhD}$ thesis, Université de Liège, 2005 .

G.-B. Stan, A. Hamadeh, R. Sepulchre, and J. M. Gonçalves. Output synchronization in networks of cyclic biochemical oscillators. In American Control Conference $(A C C)$, Times Square, New York City, USA, July 11-13 2007.

S. H. Strogatz. Synch: the emerging science of spontaneous order. Hyperion, 2003.

X. Xu and P. J. Antsaklis. Stabilization of second-order LTI switched systems. In IEEE Conference on Decision and Control (CDC), pages 1339-1344, Phoenix, Arizona, USA, 1999. 